\title{
Intra-aortic balloon pump. A cheap device to protect CHIP?
}

\author{
Giuseppe Andò, Roberta Manganaro, Giovanni Andò \\ Department of Clinical and Experimental Medicine, University of Messina, Messina, Italy
}

Adv Interv Cardiol 2020; 16, 1 (59): 10-14

DOI: https://doi.org/10.5114/aic.2020.93907

Variables that contribute to elevated risk during percutaneous coronary intervention $(\mathrm{PCl})$ have been well defined and can be categorized into two major groups: 1) patient-specific, such as advanced age, poor left ventricular function, severe comorbidities, clinical presentation with acute coronary syndrome or cardiogenic shock; and 2) procedural-specific, such as complex anatomy, involvement of left main coronary artery or major bifurcations, heavy calcifications, chronic total occlusions, last patent vessel. Clinical features make these patients at high risk for standard coronary artery bypass grafting; nonetheless, they are also at high risk of intra-procedural complications with $\mathrm{PCl}$ and, whenever they are left untreated, they face a poor prognosis. High-risk PCI remains a challenge in modern interventional cardiology.

Catheter-based devices for mechanical circulatory support (MCS) aim both to overcome the inability of the heart to provide an adequate output and to sustain blood circulation and hemodynamics (Table I). Mechanistically, left ventricular support devices unload the failing left ventricle by reducing pre-load (volume unloading), after-load (pressure unloading) or both, whereas circulatory support increases mean arterial blood pressure. The ultimate goal is to improve or maintain myocardial, cerebral, mesenteric, renal, and peripheral tissue perfusion. MCS devices are routinely used during procedures on complex, higher-risk and indicated patients (CHIP), which are currently referred to as "protected PCI". CHIP may frequently suffer intra-procedural sustained hypotension, which in turn jeopardizes coronary perfusion gradient. Loss of coronary perfusion leads to profound myocardial ischemia, which quickly depresses the already impaired left ventricle and may lead to cardiovascular collapse and cardiac arrest.

Intra-aortic balloon pump (IABP) is the simplest form of MCS (Table I). IABP aims to augment coronary and sys- temic blood flow during diastole while decreasing myocardial oxygen consumption and both left ventricular wall stress and workload. It only modestly enhances cardiac output. The demonstration of the protective role of elective IABP in protected $\mathrm{PCI}$ is still a controversial issue. Beyond historical positive data from observational studies [1], the largest randomized trial so far, the Balloon Pump-Assisted Coronary Intervention Study (BCIS-1), yielded negative results in the short term [2], but demonstrated a $34 \%$ relative reduction in all-cause mortality at a median follow-up of 51 months [3]. Despite discouraging evidence also in the setting of cardiogenic shock complicating acute myocardial infarction [4] and a class III European recommendation for routine IABP use in this setting [5], IABP is still widely used during elective complex $\mathrm{PCl}$ [6]. IABP is actually an inexpensive, readily available device that is associated with ease of use and post-implantation management and a low incidence of thrombotic and bleeding complications [7].

More complex and invasive MCS systems (Table I), such as Impella (Abiomed, Danvers, Massachusetts) devices, provide a definitely higher hemodynamic support and - in the pivotal randomized trial - yielded a reduction of post-discharge major adverse events and an improvement in ventricular function and heart failure symptoms [8]. However, Impella or even Veno-Arterial Extra Corporeal Membrane Oxygenation (VA-ECMO) devices require large-bore vascular access (Table I), which entail an appropriate learning curve and are associated with a high burden of complications [9]. Moreover, technical feasibility of vascular access remains a key issue for MCS device implantation as these patients frequently have tortuosity and extensively calcific disease in the ilio-femoral arteries that create additional challenges to the interventionalist. Recent non-randomized data, although flawed by possible unmeasured residual confounding, have raised

\section{Corresponding author:}

Prof. Giuseppe Andò, Department of Clinical and Experimental Medicine, Section of Cardiology, University of Messina, Messina, Italy, phone: +39 3473255000, e-mail: giuseppeando1975@gmail.com

Received: 4.02.2020, accepted: 13.02.2020. 


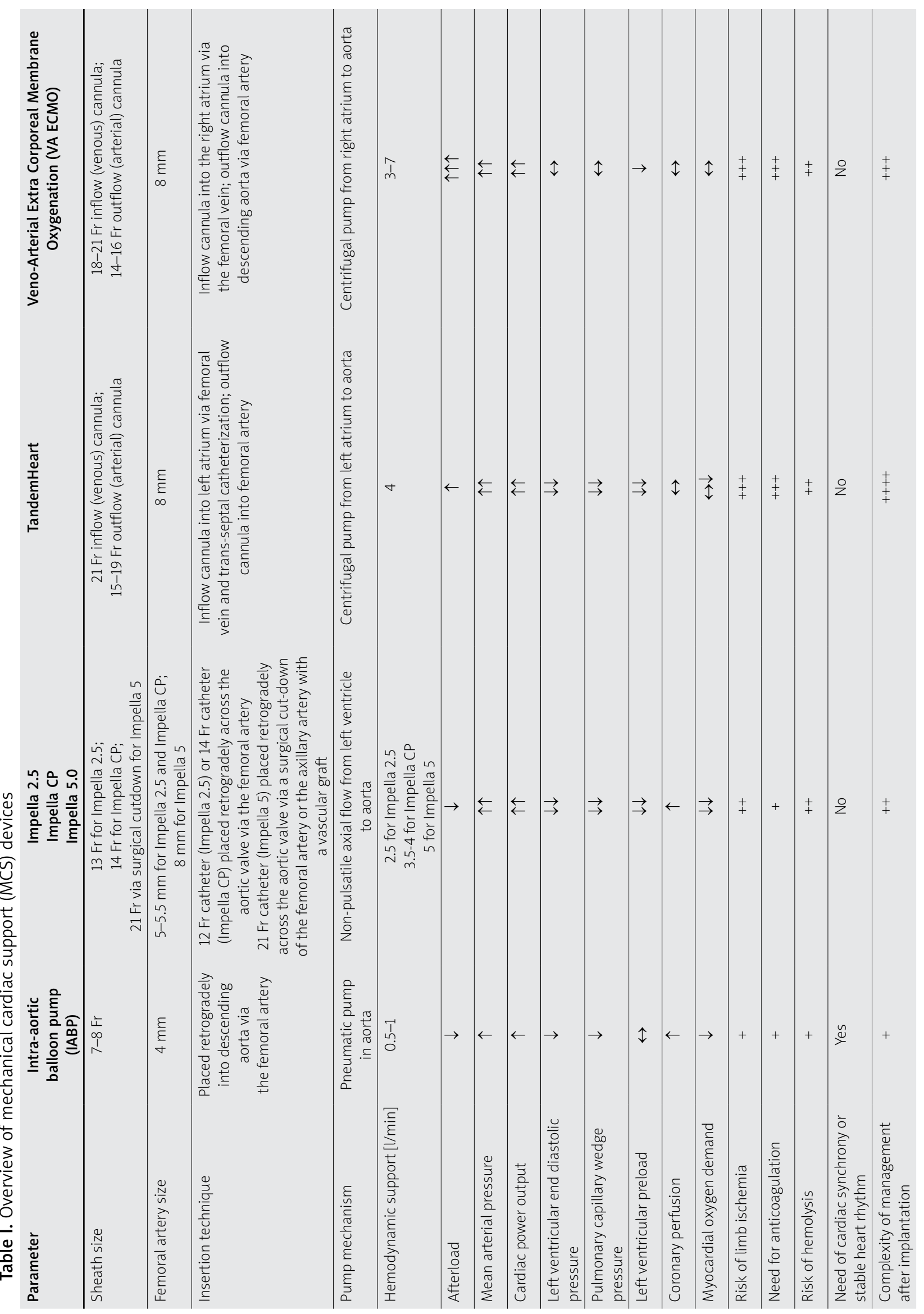


questions about the association of Impella devices with higher rates of adverse events and costs [10]. Although VA-ECMO provides full cardiopulmonary support, it may require left ventricular venting or unloading to prevent increased myocardial oxygen demand secondary to high filling pressures and volume induced by circulatory support, which can precipitate further myocardial ischemia. This imposes additional complexity on management because a second device aimed at reducing afterload (ei- ther IABP or Impella) may be needed beyond the support of an experienced cardiac perfusionist.

Basically, the unmet need in high-risk complex $\mathrm{PCl}$ is to achieve stable procedural hemodynamics with few hypotensive events, allowing for more extensive atherectomy and greater and more complete revascularization, which is expected to provide long-term benefits [11]. An illustrative case of IABP-protected PCI is presented in the Figure 1 . To this purpose, the authors of the elegant ran-
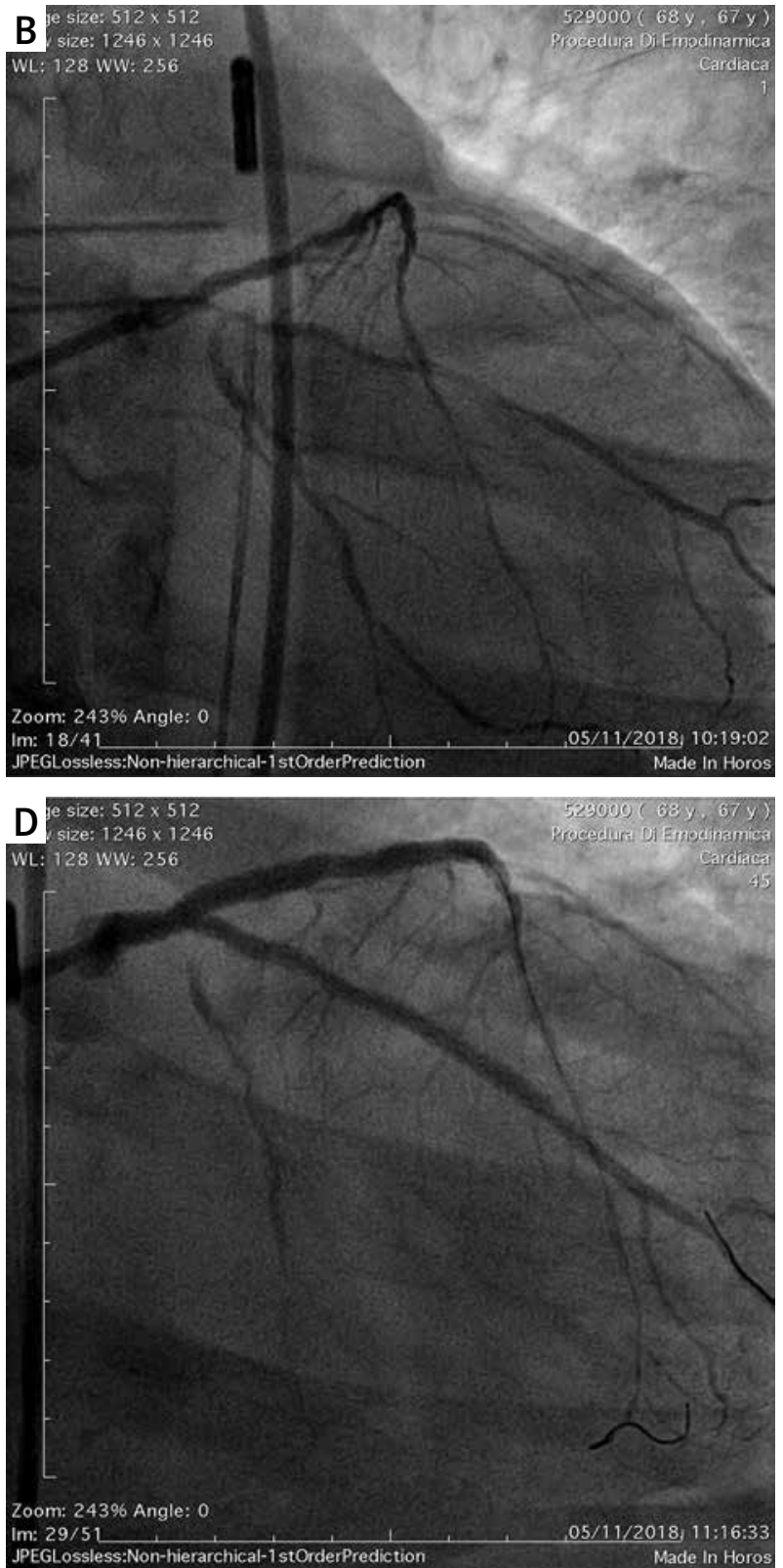
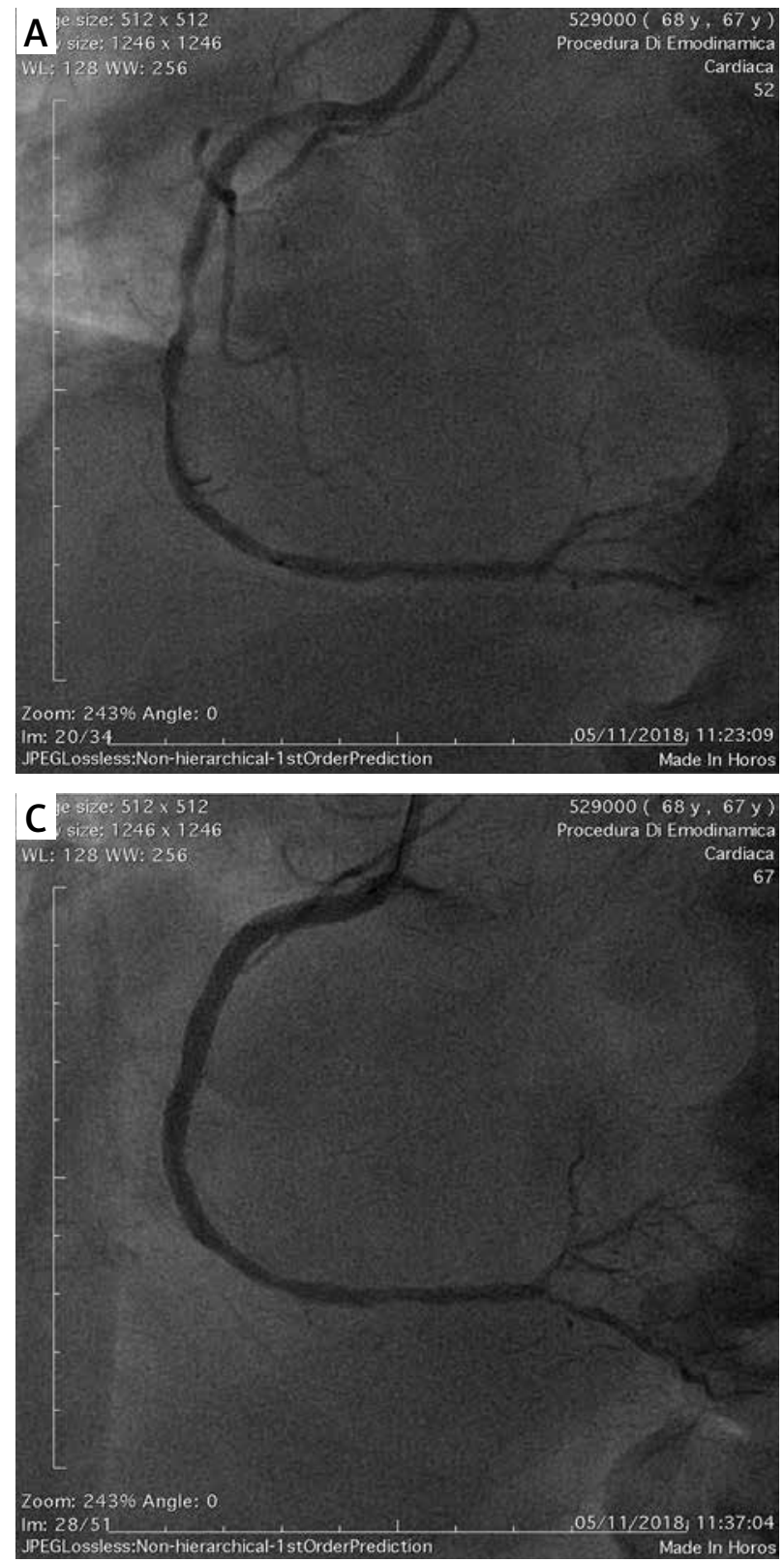

Figure 1. An illustrative example of complete revascularization with IABP-protected PCI in a diabetic 67-yearold male CHIP with stage IV chronic kidney disease, poor left ventricular function and recent pulmonary edema, having received surgical turndown. Top panels, baseline angiography demonstrating significant lesions in right coronary artery (A) and critical calcific disease involving left main bifurcation, left anterior descending and circumflex arteries (B). Bottom panels, final result after implantation of 2 long drug-eluting stents (DES) in right coronary artery $(\mathbf{C})$ and, after extensive rotational atherectomy, implantation of 2 DES in the left main bifurcation with double-kissing crush technique and 1 additional DES in left anterior descending and circumflex arteries (D) 
domized single-center study published in the present issue [12] of Advances in Interventional Cardiology/Postępy $w$ Kardiologii Interwencyjnej investigated whether IABP assistance with a $10 \mathrm{ml}$ larger than standard (MEGA) balloon provides better clinical results or, at least, an improvement in hemodynamic support in CHIP undergoing high-risk $\mathrm{PCl}$ as compared with standard IABP or with no support. Notably, patients with cardiogenic shock or recent acute coronary syndrome were excluded per the protocol; therefore the results apply only to non-emergent elective procedures.

Being small in magnitude $(n=36)$, this study did not have the power to detect any difference in hard endpoints across randomized groups, which were as high as expected (in-hospital MACE 19\%, in-hospital major bleeding 33\%, cardiovascular death during follow-up $25 \%)$. However, the authors have to be commended for having used a separate arterial line to constantly record arterial pressure in order to define a new hemodynamic composite endpoint. Interestingly, they observed a trend towards lower hypotension load and maximum hypotensive episodes with MEGA balloon, that mechanistically led to less periprocedural myocardial damage.

To observe hemodynamic parameters is indeed a crucial issue during protected $\mathrm{PCl}$ and to set hemodynamic endpoints may represent a new line of research.

Cardiac power output [(cardiac ouput $\times$ mean arterial pressure)/451] [13], expressed in watts, couples both adequacy of systemic blood flow and maintenance of appropriate blood pressure (flow domain and pressure domain of the cardiovascular system, respectively), and thus is a reliable measure of cardiac pumping function. Together with serial biochemical evaluation of arterial lactate, which expresses the inadequacy of tissue perfusion, cardiac power output can be used to define, diagnose and accurately monitor cardiogenic shock. In turn, cardiogenic shock is the natural setting to evaluate the different hemodynamic performances of MCS devices. Yet, it appears not realistic in the setting of an elective protected $\mathrm{PCl}$, as complex and risky as possible but lasting no more than a few hours, the attempt to implement such a complex evaluation that requires pulmonary artery catheterization and prolonged multiparametric observation. Beat by beat evaluation of cardiac power (PWR) is the continuous equivalent of cardiac power output (CPO) but still requires clinical validation and additional invasiveness [14].

Ultimately, hypotensive episodes demonstrated by continuous arterial pressure monitoring may represent a reasonable surrogate indicator of transient coronary and end-organ hypoperfusion. In the pivotal trial of Impella, indeed, the intra-procedural decrease in mean arterial pressure was consistent with the reduction of cardiac power output [15].

In conclusion, given the limited prospective, randomized, multicenter data with use of advanced MCS in protected $\mathrm{PCl}$ and despite the contemporary increasing use
[10], the relative role of the diverse, commercially available MCS devices in patients undergoing high-risk $\mathrm{PCl}$ is yet to be completely defined. However, we hardly can expect new conclusive evidence because of the difficulties and the ethical implications in performing randomized studies in this setting. In addition, the lack of a reliable risk tool to indicate use of MCS during $\mathrm{PCl}$ is an unmet need that warrants further research. IABP remains the easiest and fastest MCS device with the lowest rate of complications and the lowest complexity in post-implantation management. This still makes IABP irreplaceable in all those contexts in which drug therapy alone is not sufficient to support and stabilize hemodynamics and at the same time the use of more invasive devices is not feasible. A small additional IABP balloon volume is a promising option to provide a simple, cheap and effective increase in hemodynamic support that deserves confirmation in larger prospective studies. To use a separate arterial line during CHIP procedures makes it possible to constantly calculate derivative parameters that may prove useful as surrogate indicators of hemodynamic derangement in the era of modern MCS.

\section{Conflict of interest}

The authors declare no conflict of interest.

\section{References}

1. Mishra S, Chu WW, Torguson R, et al. Role of prophylactic intraaortic balloon pump in high-risk patients undergoing percutaneous coronary intervention. Am J Cardiol 2006; 98: 608-12.

2. Perera D, Stables R, Thomas M, et al. Elective intra-aortic balloon counterpulsation during high-risk percutaneous coronary intervention: a randomized controlled trial. JAMA 2010; 304: 867-74.

3. Perera D, Stables R, Clayton T, et al. Long-term mortality data from the balloon pump-assisted coronary intervention study (BCIS-1): a randomized, controlled trial of elective balloon counterpulsation during high-risk percutaneous coronary intervention. Circulation 2013; 127: 207-12.

4. Thiele H, Zeymer U, Neumann FJ, et al. Intra-aortic balloon counterpulsation in acute myocardial infarction complicated by cardiogenic shock (IABP-SHOCK II): final 12 month results of a randomised, open-label trial. Lancet 2013; 382: 1638-45.

5. Neumann FJ, Sousa-Uva M, Ahlsson A, et al. 2018 ESC/EACTS Guidelines on myocardial revascularization. Eur Heart J 2019; 40: 87-165.

6. Curtis JP, Rathore SS, Wang Y, et al. Use and effectiveness of intra-aortic balloon pumps among patients undergoing high risk percutaneous coronary intervention: insights from the National Cardiovascular Data Registry. Circ Cardiovasc Qual Outcomes 2012; 5: 21-30.

7. Rihal CS, Naidu SS, Givertz MM, et al. 2015 SCAI/ACC/HFSA/STS Clinical Expert Consensus Statement on the Use of Percutaneous Mechanical Circulatory Support Devices in Cardiovascular Care: Endorsed by the American Heart Assocation, the Cardiological Society of India, and Sociedad Latino Americana de Cardiologia Intervencion; Affirmation of Value by the Canadian Association of Interventional Cardiology-Association Canadienne de Cardiologie d'intervention. J Am Coll Cardiol 2015; 65: e7-26. 
8. O'Neill WW, Kleiman NS, Moses J, et al. A prospective, randomized clinical trial of hemodynamic support with Impella 2.5 versus intra-aortic balloon pump in patients undergoing high-risk percutaneous coronary intervention: the PROTECT II study. Circulation 2012; 126: 1717-27.

9. Schrage B, Ibrahim K, Loehn T, et al. Impella support for acute myocardial infarction complicated by cardiogenic shock. Circulation 2019; 139: 1249-58.

10. Amin AP, Spertus JA, Curtis JP, et al. The evolving landscape of impella use in the united states among patients undergoing percutaneous coronary intervention with mechanical circulatory support. Circulation 2020; 141: 273-84.

11. Dangas GD, Kini AS, Sharma SK, et al. Impact of hemodynamic support with Impella 2.5 versus intra-aortic balloon pump on prognostically important clinical outcomes in patients undergoing high-risk percutaneous coronary intervention (from the PROTECT II randomized trial). Am J Cardiol 2014; 113: 222-8.

12. Zelias A, Zajdel W, Malinowski K, et al. Circulatory support with larger volume intra-aortic balloon pump vs. standard volume or no-balloon pump during high-risk percutaneous coronary interventions. A randomized study. Adv Interv Cardiol 2020; 16: 30-40.

13. Fincke R, Hochman JS, Lowe AM, et al. Cardiac power is the strongest hemodynamic correlate of mortality in cardiogenic shock: a report from the SHOCK trial registry. J Am Coll Cardiol 2004; 44: 340-8.

14. Rimehaug AE, Skogvoll E, Aadahl P, et al. Minimally invasive beat-by-beat monitoring of cardiac power in normal hearts and during acute ventricular dysfunction. Physiol Rep 2016; 4 e12989.

15. Kovacic JC, Kini A, Banerjee S, et al. Patients with 3-vessel coronary artery disease and impaired ventricular function undergoing $\mathrm{PCl}$ with Impella 2.5 hemodynamic support have improved 90-day outcomes compared to intra-aortic balloon pump: a substudy of the PROTECT II trial. J Interv Cardiol 2015; 28: 32-40. 